\title{
Shear Stress in Torsion of Circular Shafts-not a True Stress, but an Equivalent Stress
}

\author{
Jian GUO ${ }^{1, a}$, Wen-Ba HAN ${ }^{2, b}$, Gang WANG ${ }^{c}$ \\ ${ }^{1,2}$ Panzhihua University, No. 10 Airport Road, Eastern District, Panzhihua City, 617000, Sichuan \\ Province, P.R. China \\ apzh_gj@163.com , 'caibingqing1948@yahoo.com.cn, '16160100@qq.com
}

Keywords: Shear Stress, Equivalent Stress, Plane Assumption, Equilibrium.

\begin{abstract}
The present research argues that the shear stress of shaft in torsion is not a true stress but an equivalent stress, based on the phenomena that the stress disproves the plane assumption, that the body part in torsion is unable to maintain equilibrium, and that shear stress on the longitudinal section drawn from reciprocal theorem is not only self-contradictory, but also in conflict with Newton's third law. Namely, the angle strain generated by torque equals that produced by shear stress. The above phenomena are caused by equivalent stresses.
\end{abstract}

\section{Introduction}

Shear stress is indispensable in designing the torsion of circular shafts in engineering. However, despite the use of the finite element method, which is precise, the shafts often break or lack in rigidity, which has frequently led to accidents and resulted in great loss. The reason lies in the false belief that there is true shear stress in the body of torsion. This fundamental error has mistaken the true shear stress for an equivalent stress in calculation; hence, it constitutes the principle reason for the breaking of shafts and the inadequacy of rigidity.

The present paper will illustrate that the shear stress in torsion of shafts is not a true stress but an equivalent stress from the following perspectives: 1 . the unequilibrium in torsion of circular shafts; 2 . the self-contradiction of torsional shear stresses and 3. the contradiction between torsional shear stresses and the Newton's third law.

\section{The Torsional Shear Stress Disproving the Plane Assumption}

In the deduction of the torsional shear stress, a basic assumption is that during the deformation, cross sections still remain planes without the change in form and size $[5,10,11]$. If this assumption is disproved, it follows that the formula deducted from it is false.

Fig. 1(a) shows the distribution of the shear stress on the cross section and the vertical section of the round shaft under the internal torque $M_{\mathrm{n}}$. A wedge is formed by the two intersecting sections through the axis oo' as shown in Fig. 1(b).

The stress distribution of the wedge, as shown in Fig. 1(b), is drawn from the torsion deformation formula and the theorem of conjugate shear stresses. To make it clearer, abcd of the square is marked in Fig. 1(c) so as to study the deformation of the square[2, 3]. From the principle stress formula of the pure shear $[1,4,7,8]$, we get $\sigma_{1}=\tau, \sigma_{3}=-\tau$. When the principle stress acts on the two diagonals ac and bd, ac is tensioned so that a moves to a' and c to c' along the diagonal. In the same way, bd is compressed so that $b$ moves to $b^{\prime}$ and $d$ to $d^{\prime}$. It could be seen that under the shear stress, the square has been turned into a lozenge. Obviously, a' is not on the original cross section o'ab while c' is not on ocd, as shown in Fig. 1(c). The cross section is slanted and it does not remain the plane it was. The elastic theory is contradictory with itself. Therefore, the shear stress formula and the deformation formula which are deducted from it are false.

When the thin-wall circular tube is twisted as shown by Fig. 2(a), $m m$ and $n n$, the two parallel circumference lines which are perpendicular to the axis $x$, still remain perpendicular to the axis $x$. In this 
case, the cross section remains the plane with only a change of angle, and the plane assumption is valid. Only torque can account for it because if it is due to the internal shear stress, the effect will be different as shown by Fig. 2(b): instead of being perpendicular to the axis $x$, circumference lines $m m$ and $n n$ turn to be ellipses $m^{\prime} m^{\prime}$ and $n ' n$ ' slanting to the axis $x$. Thus we can see that, both theories and experiments proves that the theory of shear stress in torsion cannot guarantee the validity of the plane assumption.

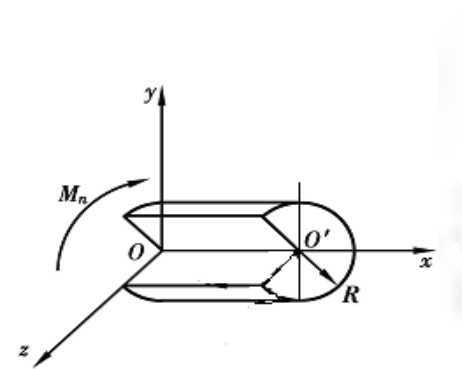

(a)

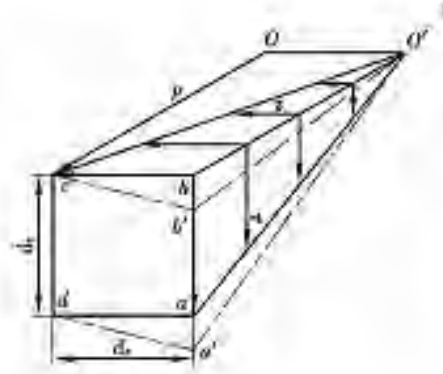

(b)

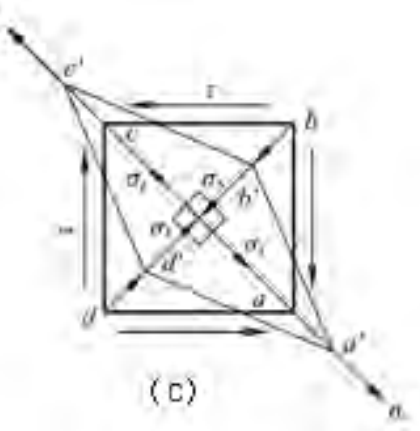

(c)

Fig. 1 The Shear Stress in Torsion of Circular Shaft Denies the Plane Assumption

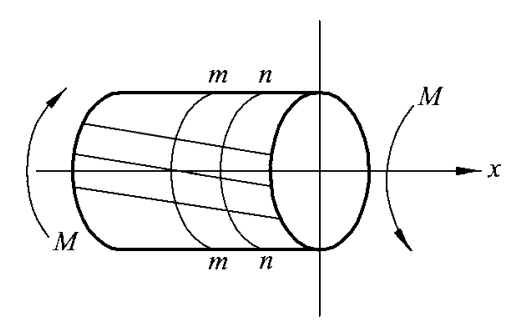

(a)

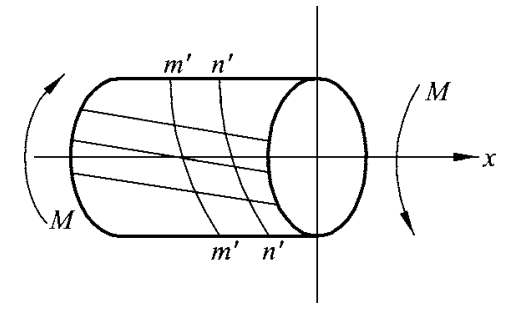

(b)

Fig. 2 The Torsional Shear Stress Violates the Plane Assumption in Torsion of the Thin-wall Circular Tube

\section{The Unequilibrium in the Torsion of the Cylinder}

\section{Example 1}

As shown in Fig.3(a), the cylinder with the radius of $R$, which three force couples act on, is in equilibrium.

Let $M_{A}=M_{C}=M$, with the equilibrium condition $M_{B}=M_{A}+M_{C}=-2 M$, its torque should be what is shown in Fig. 3(b).

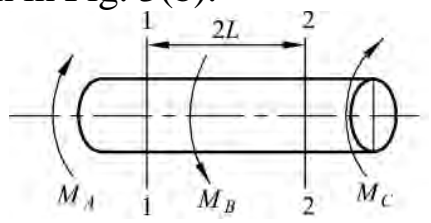

(a)

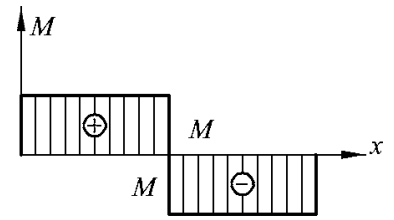

(b)

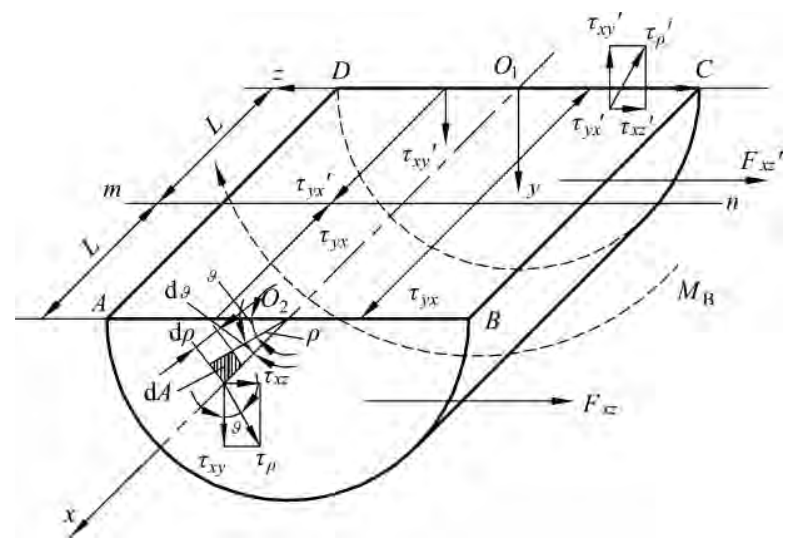

(c)

Fig. 3 The Torsioned Body of the Cylinder which Three Force Couples Act on is in Unequilibrium

At the place distant from the couple force $M_{B}$ (as is required by Saint Venant's Principle), a section of equivalence shaft between the section of 1-1 and 2-2 is intercepted perpendicularly and is cut apart along the central line to study the equilibrium of the lower half of the cylinder, on which the couple force $M_{B}$ acts as shown in Fig 3(c). 
$\tau_{\rho}$ is the torsional shear stress which acts on the end plane $(A B)$. Break $\tau_{\rho}$ up into two stress components $\tau_{\mathrm{xz}}$ and $\tau_{\mathrm{xy}}$, which are perpendicular to each other. Assume the angle between $\tau_{\rho}$ and $\tau_{\mathrm{xy}}$ is $\vartheta$, we get

$$
\begin{gathered}
\tau_{\mathrm{xy}}=\tau_{\rho} \cos \vartheta . \\
\tau_{\mathrm{xz}}=\tau_{\rho} \sin \vartheta .
\end{gathered}
$$

The resultant force $F_{\mathrm{xy}}$ and $F_{\mathrm{xz}}$ from the action of $\tau_{\mathrm{xy}}$ and $\tau_{\mathrm{xz}}$ on the half-circle section $(A B)$ :

$$
F_{\mathrm{xy}}=\int_{A B} \tau_{\mathrm{xy}} d A=\int_{A B} \tau_{\rho} \cos \vartheta \mathrm{d} A
$$

Within $(A B)$, calculating the integral using polar coordinates and assuming $\rho$ the radius to the center $O$, we get micro area:

$$
\mathrm{d} A=\mathrm{ds} d \rho
$$

and micro-arc:

$$
d s=\rho d \vartheta \text {. }
$$

The torsional shear stress at any point on the cross section of the cylinder:

$$
\tau_{\rho}=\frac{M_{n} \rho}{I_{P}}
$$

Substituting Eq.(b) and Eq.(3), Eq.(a) becomes that $F_{\mathrm{xy}}=\int_{0}^{\pi} \cos \vartheta d \vartheta \int_{0}^{R} \frac{M}{I_{P}} \rho^{2} d \rho=0$.

The resultant force $F_{x y}$ from the action of $\tau_{\mathrm{xy}}$ on the half-circle section $\mathrm{AB}$ is zero. The resultant force $F_{x z}$ from the action of $\tau_{x z}$ on the half-circle section $(A B)$ :

$$
F_{x z}=\int_{(A B)} \tau_{\rho} \sin \vartheta d A=\int_{0}^{\pi} \sin \vartheta d \vartheta \int_{0}^{R} \frac{M}{I_{P}} \rho^{2} d \rho=\frac{2 M}{3 I_{P}} R^{3} .
$$

Substituting $I_{P}=\frac{\pi}{2} R^{4}$, Eq.(d) becomes that

$$
F_{x z}=\frac{4 M}{3 \pi R} \text {. }
$$

Using the same method, we get the shear stress on the cross section $(C D): F_{x y}^{\prime}=0 ; F_{\mathrm{xz}}^{\prime}=\frac{4 M}{3 \pi R}$.

Note: $F_{\mathrm{xz}}$ and $F_{\mathrm{xz}}^{\prime}$ being the same in strength and direction, the resultant force:

$$
\sum Z=F_{\mathrm{xz}}+F_{x z}^{\prime}=\frac{8 M}{3 \pi R} \text {. }
$$

Eq.(h) indicates that semicolumn $(A B)$ moves along the axis $z$, which is not what really happens. Meanwhile, $F_{x z}$ generates moment on the axis $y$ :

$$
\sum M_{y}=F_{\mathrm{xz}}(2 L)=\frac{8 M L}{3 \pi R}
$$


Eq.(i) shows that semicolumn twists around axis $y$, which is obviously contradictory with the truth.

Some people attempt to explain the contradiction by stress concentration, as shown in 4(a). They maintain that in the area of $\Delta x$ acted on by the force couple $M_{B}$ within the plane $(A B C D)$, only the resultant force $F_{\mathrm{yz}}$, which is composed of the shear stress $\tau_{\mathrm{yz}}$, can reach the equilibrium with the resultant force $\sum \mathrm{z}$, composed of $F_{\mathrm{xz}}$ and $F_{\mathrm{xz}}^{\prime}$.

If $\tau_{\mathrm{yz}}$ existed, there would be new unequilibrium: the existence of $\tau_{\mathrm{yz}}$ would break the equilibrium of $A b n ' m^{\prime}$, the front half of the cylinder which is intercepted perpendicularly at the point of $\Delta x / 4$ in the area of $\Delta x / 2$ in front of the acting line mn of moment $M_{B}$. As shown in Fig. 4 (b), on the half-circle section of $m^{\prime} n$ ', exists a resultant force $F_{\mathrm{xz}}$, which is in equilibrium, equal in magnitude and opposite in direction, with the resultant force $F_{\mathrm{xz}}$ on the half-circle section $(A B)$; however, there is no force to balance the resultant force $F_{\mathrm{xz}}$ composed of shear stress $\tau_{\mathrm{yz}}$ that acts on $\Delta x / 4$.

Only under the condition that $\tau_{\mathrm{yz}}=0$, the equilibrium of the semicolumn $\left(A b n^{\prime} m^{\prime}\right)$ can be achieved. However, neither through the methods of Elastic Mechanics nor through those of material mechanics can the shear stress $\tau_{\mathrm{yz}}$ be found in area of $\Delta x$ within the moment acting body. It proves that stress concentration cannot be used to explain the unequalibrium in the torsion of cylinder.
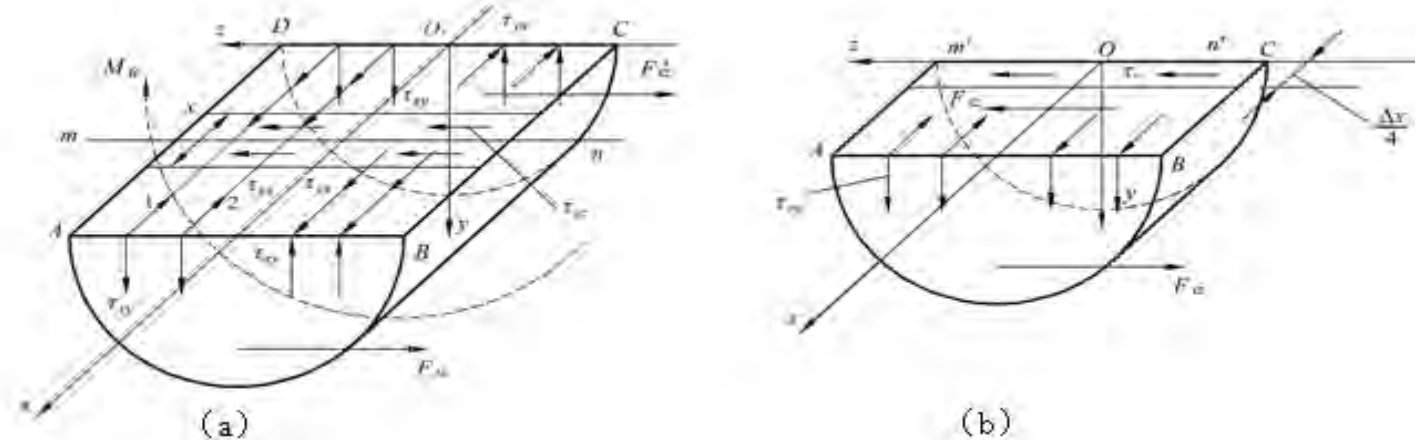

Fig. 4 The Stress Concentration can not Explain the Unequilibrium of Torsional Body

\section{Example 2}

As shown in Fig.5 (a), the round shaft acted on by couple $m$ is divided along diameter $d$, and on the longitudinal section, owing to the shear stress reciprocal theorem ${ }^{[6.9]}$, there will exist $\tau^{[1.7]}$, whose direction is shown in Fig. 5 (b). The resultant force of shear stress on the longitudinal section $O O O^{\prime} C D$ and that on $O O^{\prime} B A$ make up a moment to $y$-axis, which is not balanced with others, because there is no stress on both ends $(A D)$ and $(B C)$.

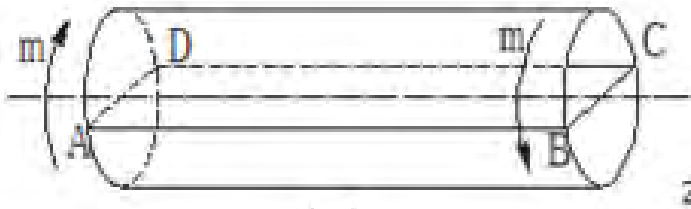

(a)

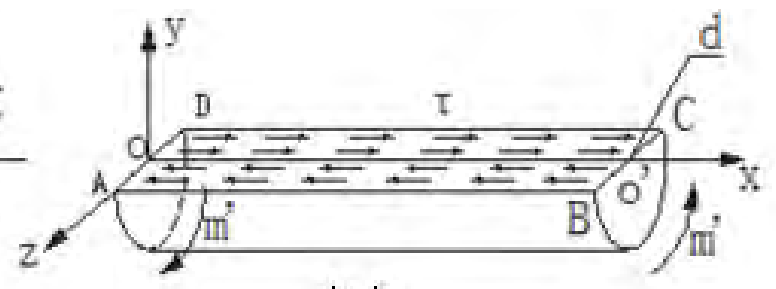

(b)

Fig. 5 Semi-round Shaft is in Unequalibrium Under the Action of Two Balanced Couples

\section{Contradiction between Shear Stress Reciprocal Theorem and Newton's Third Law}

What is shown in Fig. 6 (a) is the uniform bar that has been cut. After it is cut vertically from the position $m n$ in the middle part, the shear stress on its section is shown in Fig. 6 (b). 
On the surface $(A B C E)$ of the straight bar that bears the torque $M_{n}$ and has been cut, according to the shear stress reciprocal theorem, there exists $\tau_{\mathrm{yx}}$ that is caused by $\tau_{x y}$. From the torque Fig.7 we can see that, along the $x$ direction of the bar, torque is a constant, therefore, the absolute value of shear stress $\tau_{\mathrm{yx}}$ on line $11^{\prime}$ or 22 ' that have equal distance from the $z$ coordinate should be equal. The present problem is where we shall draw $\tau_{\mathrm{yx}}$ as far as to? Someone say, $\tau_{\mathrm{yx}}$ should be drawn as far as to line $m n$, and $\tau_{y x}^{\prime}$ to there, too. By this way of drawing, a result violating the Newton's third law shall appear: according to the shear stress reciprocal theorem, if $\tau_{\mathrm{xy}}^{\prime \prime}$ is applied on section $(m n)$, on section $\left(m^{\prime} n^{\prime}\right)$ there will be $\tau_{\mathrm{xy}}^{\prime \prime \prime}$. Existence of $\tau_{\mathrm{xy}}^{\prime \prime \prime}$ and $\tau_{\mathrm{xy}}^{\prime \prime}$ that are same value and same direction violates the Newton's third law. The Newton's third law cannot be wrong; it is only possible that there exists limitation in the shear stress reciprocal theorem.

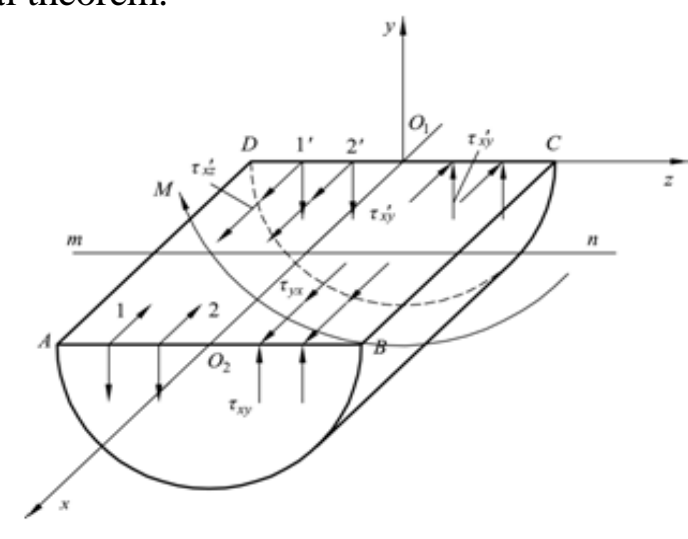

(a)

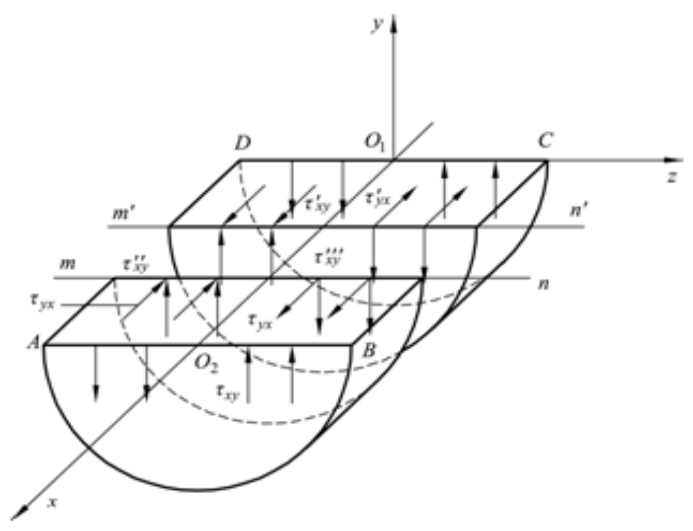

(b)

Fig. 6 Contradiction between Shear Stress Reciprocal Theorem and Newton's Third Law

\section{Self-contradiction of the Shear Stress Reciprocal Theorem}

From above we can see that, $\tau_{\mathrm{yx}}$ shear stress shall not be drawn as far as to the line $m m$, and it shall pass the line. This practice conforms to the derivation conditions of the shear stress reciprocal theorem. The differential balanced bodies chosen for derivation of the shear stress reciprocal theorem are randomly chosen in the body given a force, including those differential balanced bodies that are differential bodies on the action line of external force and external torque.

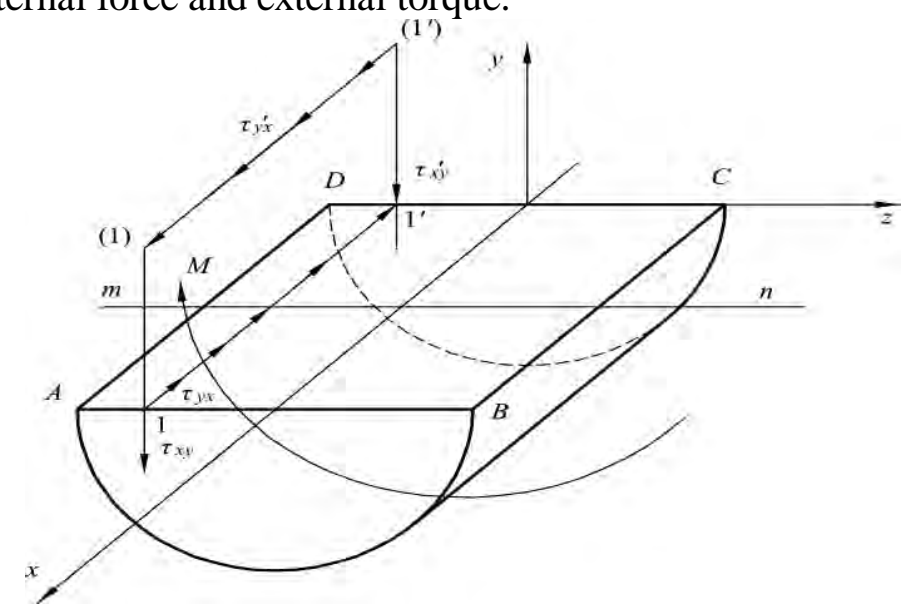

Fig. 7 Shear Stress Reciprocal Theorem Denies by Itself the Shear Stress on Longitudinal Section

In Fig.7 is drawn the shear stress that is deduced through shear stress reciprocal theorem from $\tau_{\mathrm{yx}}$ and $\tau_{x y}$, and is between sections mutually vertical. The arrow of line 1-1' indicates $\tau_{\mathrm{yx}}$ that is caused by 
torsional shear stress $\tau_{x y}$, and arrow of line (1)-(1') indicates $\tau_{y x}^{\prime}$ caused by $\tau_{x y}^{\prime}$. Originally, line 1-1' and line (1)-(1') coincide together, and separate from each other to make it clearer. Obviously, any point on the line 1-1' will bear shear stresses that are equal in magnitude and opposite in direction, i.e. on any point the joint shear stress shall be zero: $\tau_{\mathrm{yx}}-\tau_{y x}^{\prime}=0$. Similarly we can see that, the shear stress resultant force of any points on the section $(A B C D)$ will be zero, i.e. there is no shear stress on the section $(A B C D)$.

\section{Conclusion}

1. Through the analysis of above four contradictory examples of the elasticity theory, we can draw the following conclusions:

(1) Pure torsional shear stresses of the round shaft deduced by the present elasticity theory is not a real shear stress, but an equivalent shear stress.

(2) All contradictions above are caused by the mistake of regarding equivalent shear stresses regarded as true stresses.

(3) In order to solve the above fundamental contradictions of elasticity theory, what we can do is to revise the zero stress torque (the limit of torque acted on the unit area) identified by present elasticity theory, and convert it into non-zero stress torque ${ }^{[11]}$.

2. Present elasticity theory cannot solve the above contradictions of unequilibrium, while equilibrium is the foundation of the theory, because the present elasticity theory believes that stress torque(the limit of torque acted on the unit area) is zero, but actually it is not zero. From the above analysis, we can draw the following conclusions on the new elasticity(stress torque) theory:

(1) There is no shear stress on pure torsion cross-section, and the torque on the ring is produced by torsion stress torque(the limit of torque acted on the unit area), instead of shear stress;

(2) When the material yields, stress torque and the angle strain produced by the equivalent shear stress(not stress, just means how much shear stress it equals when angle strain that is same as the torsion stress torque is produced) should be equal.

\section{References}

[1]Fan Qinshan, Yin Yajun, Mechanics of Materials, Tsinghua University Press, Beijing, 2005.

[2]Han Wenba, Huang Shuanghua, Nonzero Stress Torque Elastic Theory, Chongqing University Press, Chongqing, 2013.

[3]Han Wenba, Liu Dabin, Cai Bingqing and Han Xiaodong, Stress on the unit body is not inclined section on particle equilibrium stress, China Engineering Science. 11(2005)42-47.

[4]Huang Yan, Engineering Elasticity, Tsinghua University Press, Beijing, 1982.

[5]Liu Hongwen, Mechanics of Materials, Higher Education Press, Beijing, 2000.

[6]Qian Weichang, Ye Kaiyuan, Elasticity, Science Press, Beijing, 1956.

[7]San Huizhu, Mechanics of Materials, Higher Education Press, Beijing, 2004.

[8]San Huizhu, Xie Chuanfeng, Mechanics of Materials, Higher Education Press, Beijing, 2004.

[9]Zhao Jiujiang, Zhang Shaoshi, Wang Chunxiang, Mechanics of Materials, Harbin Institute of Technology Press, Harbin, 1987.

[10]Zhao Jiujiang, Zhang Shaosih and Wang Chunxiang, Mechanics of Materials, Harbin Institute of Technology Press, Harbin, 2002.

[11]Zhang Rusan, Wang Tianming, Mechanics of Materials, China Building Industry Press, Beijing, 2002. 